\title{
Reduction in milk fat globule-EGF factor 8 inhibits triple-negative breast cancer cell viability and migration
}

\author{
YONG YANG，JIEBAO LI，QI SONG，KONGJUN ZHU，XIAOCHENG YU，YE TIAN and JIAHENG ZHANG \\ Department of Breast and Thyroid Surgery, Wuhan No. 1 Hospital Affiliated to Tongji Medical College, \\ Huazhong University of Science and Technology, Wuhan, Hubei 430022, P.R. China
}

Received April 16, 2017; Accepted January 29, 2018

DOI: $10.3892 /$ ol.2019.9968

\begin{abstract}
Milk fat globule-EGF factor 8 (MFG-E8) has been demonstrated to be associated with the progression and metastasis of breast cancer, although the underlying mechanisms remain undefined. The aim of the present study was to explore the role of MFG-E8 in human breast cancer and examine the underlying molecular mechanisms. Reverse transcription-quantitative polymerase chain reaction analysis was used to evaluate the expression levels of MFG-E8 in human breast carcinoma cell lines. An MFG-E8 small interfering RNA lentiviral vector was constructed and transfected into MDA-MB-231 cells. The results indicated that the in vitro silencing of MFG-E8 significantly inhibited the viability, invasion and migration of breast cancer cells. By using a flow cytometric approach, the knockdown of MFG-E8 was revealed to significantly induce cell cycle arrest at the $\mathrm{G} 2 / \mathrm{M}$ phase and cell apoptosis. Furthermore, the downregulation of MFG-E8 induced the activation of apoptosis-associated proteins, and inhibited the expression of matrix metalloproteinase and epithelial-mesenchymal transition-associated proteins. Collectively, the results of the present study emphasize the importance of MFG-E8 deregulation in mammary carcinogenesis and its potential use as a biomarker for the diagnosis of breast carcinomas.
\end{abstract}

\section{Introduction}

Breast cancer is one of the most malignant cancer types and the global leading cause of cancer-associated mortality in women (1). The etiology of breast cancer is multi-factorial and there are various associated risk factors, including high breast density, a late first birth and education-associated risk

Correspondence to: Dr Jiaheng Zhang, Department of Breast and Thyroid Surgery, Wuhan No. 1 Hospital Affiliated to Tongji Medical College, Huazhong University of Science and Technology, 215 Zhongshan Avenue, Wuhan, Hubei 430022, P.R. China

E-mail: liufamily0102@sina.com

Key words: milk fat globule-EGF factor 8, human breast cancer, mammary carcinogenesis factors (2). In recent years, the incidence of breast cancer in women has consistently increased (3). The primary causes of mortality in patients with breast cancer result from excessive proliferation and metastasis of cancer cells (4). Thus, a greater comprehension of the mechanisms underlying breast cancer has become a matter of urgency.

Milk fat globule-EGF factor 8 (MFG-E8), also known as lactadherin (5), is a $46 \mathrm{kDa}$ glycoprotein originally found in milk and mammary epithelial cells (6). The protein contains a signal sequence for secretion, two $\mathrm{N}$-terminal epidermal growth factor domains, and two C-terminal discoidin domains with homology to the $\mathrm{C} 1$ and $\mathrm{C} 2$ domains found in blood clotting factors $\mathrm{V}$ and VIII $(7,8)$. MFG-E8 is primarily produced by macrophages and dendritic cells (9), but is expressed in several cell types, including mammary epithelial, myoepithelial (10), macrophage (11), dendritic (12), endothelial (13), intestinal and retinal epithelial (14) cells. The protein exerts various effects on cellular proliferation, differentiation, apoptosis, migration and invasion (15). BA46, also known as MFG-E8, has been studied as a potential marker for breast cancer as it was identified in the circulation of patients with breast cancer, but not in healthy subjects $(7,16,17)$. There is also considerable interest in BA46 as a potential target for breast cancer therapy, because it is expressed in human breast carcinoma $(7,18,19)$, and radioconjugates of monoclonal antibodies that specifically recognize BA46 have successfully targeted human breast tumors transplanted into mice (20-22).

In recent decades, numerous studies have examined the mechanisms of MFG-E8 and breast cancer. These studies determined that MFG-E8 was significantly expressed in systemic lupus erythematosus (23), lung fibrosis (24), breast cancer (19) and melanoma $(25,26)$. Several different functions of MFG-E8 have been proposed in breast cancer cell lines. Yang et al (27) identified the expression and function of MFG-E8 in different breast cancer subtypes using a microarray analysis of laser capture-microdissected tissues and in situ analysis. As MFG-E8 expression levels were decreased in estrogen receptor (ER)-positive and receptor tyrosine-protein kinase erbB-2 (erbB2)-positive human breast cancer, it was concluded that MFG-E8 may exert an inhibitory function in these cancer types (27). In contrast, MFG-E8 was identified to be highly expressed in triple-negative [ER//progesterone receptor $\left.(\mathrm{PgR})^{-/ e r b B 2}\right]$ breast cancer $(\mathrm{TNBC})$ cell lines and patient sera compared with non-triple-negative cell 
lines including T47D, ZR75, MCF7, BT474 and SKBR3 and compared with basal-like human breast cancer, respectively $(27,28)$. These findings underscore the putative value of MFG-E8 as a potential biomarker and therapeutic target for breast carcinoma, although further research is required to understand the functional properties of MFG-E8 in breast carcinoma (15). In the present study, to determine the effect of MFG-E8 on the malignant and metastatic potential of TNBC cells, biological methods were used to investigate the function of MFG-E8 in MDA-MB-231 cells in vitro. Cell viability, migration, invasion and apoptosis were affected by the downregulation of MFG-E8 in human breast cancer cells.

\section{Materials and methods}

Cell culture. The human breast carcinoma cell line lines Hs578Bst (non-breast cancer cell line) (29), Hs 578T, MCF-7, ZR-75-30, T47D and MDA-MB-231 were purchased from the American Type Culture Collection (ATCC, Manassas, VA, USA). All cell lines were cultured in Dulbecco's modified Eagle's medium (DMEM) supplemented with $10 \%$ fetal bovine serum (FBS) and $1 \%$ penicillin/streptomycin at $37^{\circ} \mathrm{C}$ in an atmosphere with $5 \% \mathrm{CO}_{2}$, as recommended by ATCC.

RNA isolation and RT-qPCR analysis. RNA extraction was performed using TRIzol reagent (Life Technologies; Thermo Fisher Scientific, Inc., Waltham, MA, USA) and reverse transcribed to complementary DNA (cDNA) using the PrimeScript $^{\mathrm{TM}}$ RT reagent kit (Takara Bio, Inc., Otsu, Japan). For RT-qPCR, cDNA was mixed with the appropriate primers and the SYBR-Green Super mix (Kapa Biosystems, Inc., Wilmington, MA, USA) and run on the CFX96 Real-Time system (Bio-Rad Laboratories, Inc., Hercules, CA, USA). All mRNA data were normalized to the expression of GAPDH. The primers used in the present study were as follows: Human MFG-E8 forward, 5'-GTAACTTTGGCTCTGTCC-3' and reverse, 5'-GTTCTTCTTGTGGGAGTG-3'; human GAPDH forward, 5'-CCACTCCTCCACCTTTG-3' and reverse, 5'-CAC CACCCTGTTGCTGT-3'. The procedure for RT-qPCR included $5 \mathrm{~min}$ at $99^{\circ} \mathrm{C}$, followed by 40 cycles of $15 \mathrm{sec}$ at $94^{\circ} \mathrm{C}, 30 \mathrm{sec}$ at $59^{\circ} \mathrm{C}$ and $45 \mathrm{sec}$ at $72^{\circ} \mathrm{C}$. The $2^{-\Delta \Delta C q}$ method was used to calculate the relative expression of MGF-E8 (30).

Lentivirus production and oligonucleotide transfection. Lentivirus containing MFG-E8 short hairpin RNA1 and RNA2 (shRNA1, shRNA2) or scrambled oligonucleotides were obtained from Wuhan Hualian Branch Biotechnology Co., Ltd. (Wuchang, Wuhan, China). The cells were divided into four groups including the blank control group (Control), the scramble group (pSi-Scramble), the shRNA1 group, and the shRNA2 group. The target sequences for the MFG-E8 shRNA were as follows: MFG-E8 shRNA2, forward, 5'- tat gGGACTGGCAGCAGTAAGATCTTTCAAGAGAAGATC TTACTGCTGCCAGTCCTTTTT-3' and reverse, 5'- aattAA AAAGGACTGGCAGCAGTA AGATCTTCTCTTGAA AGATCTTACTGCTGCCAGTCC-3'. The cells were inoculated into 6-well plates at a concentration of $3 \times 10^{5}$ cells $/ \mathrm{ml}$ $\left(1.2 \times 10^{6}\right.$ per well $), 4 \mathrm{ml}$ of complete medium was added, mixed well, and the plates were placed in a $\mathrm{CO}_{2}$ incubator overnight at $37^{\circ} \mathrm{C}$. Two $\mu \mathrm{g}$ of the plasmid (pGMLV-SC5RNAi;
Genomeditech, Shanghai, China) to be transfected was diluted in $100 \mu \mathrm{l}$ of serum-free medium to make solution A and $25 \mu \mathrm{l}$ of Lipofectamine ${ }^{\circledR} 2000$ was diluted in $100 \mu \mathrm{l}$ of serum-free medium to make solution B. Solutions A and B were mixed and agitated for $30 \mathrm{~min}$ at $18-21^{\circ} \mathrm{C}$ (room temperature), then added to the cells for incubation in 6-well plates at $37^{\circ} \mathrm{C}$ for $6 \mathrm{~h}$. Following transfection, the cells were washed twice with serum-free medium and cultured for 3 days, after which the protein expression in cells was evaluated. A total of $50 \mathrm{nM}$ pSi-MFG-E8 and $20 \mathrm{nM}$ pSi-Scramble (Wuhan Hualian Branch Biotechnology Co., Ltd.) were diluted in Opti-L-15 reduced serum medium (Gibco; Thermo Fisher Scientific, Inc., Waltham, MA, USA); the diluted plasmid DNA and Lipofectamine 2000 were then mixed at a ratio of 1:2.5 and added to MDA-MB-231 cells.

MTT assay. Cell viability was measured using a MTT assay. MDA-MB-231 cells ( $2 \times 10^{5}$ cells per well) were plated in 96-well plates, allowed to adhere overnight and transfected with pSi-Scramble or pSi-MFG-E8 as aforementioned. Untreated MDA-MB-231 cells were used as the control. After transfection for 24,48 and $72 \mathrm{~h}, 20 \mu \mathrm{l}$ MTT $(5 \mathrm{mg} / \mathrm{ml})$ was added and the plates were incubated for a further $4 \mathrm{~h}$ at $37^{\circ} \mathrm{C}$. Subsequently, $150 \mu 1 \mathrm{DMSO}$ was added to dissolve the formazan crystals. The optical density (OD) was detected at $490 \mathrm{~nm}$ using a microplate spectrophotometer. The cell survival percentage was calculated as follows: (OD sample/OD control) $\mathrm{x} 100 \%$.

Cell cycle assay. The cell cycle distribution was determined using flow cytometry. MDA-MB-231 cells were fixed in 70\% cold ethanol at $4^{\circ} \mathrm{C}$ for $30 \mathrm{~min}$. Following fixation, the cells were washed twice with PBS and collected by centrifugation at $3,000 \mathrm{xg}$ for $30 \mathrm{sec}$ at $4^{\circ} \mathrm{C}$. The collected pellets were suspended and incubated in PBS containing $20 \mu \mathrm{l} / \mathrm{ml}$ of propidium iodide (PI), $0.2 \%$ Triton X-100, and $40 \mu \mathrm{g} / \mathrm{ml}$ RNaseA at $4^{\circ} \mathrm{C}$ for at least $30 \mathrm{~min}$. Finally, the cell cycle phase distribution was assessed using a flow cytometer.

Tumor cell migration and invasion assays. Transwell chambers (8- $\mu \mathrm{m}$ diameter, 24-well format) (Corning Incorporated, Corning, NY, USA) were used in the assay. For the cell invasion assay, the internal surface of each polycarbonate membrane was coated with Matrigel ${ }^{\mathrm{TM}}(30 \mu \mathrm{g})$ for $30 \mathrm{~min}$ at $37^{\circ} \mathrm{C}$ for gel formation (however, for the cell migration assay, the polycarbonate membrane was not coated with Matrigel) and then blocked with $500 \mu \mathrm{l}$ serum-free DMEM media. MDA-MB-231 cells ( $1 \times 10^{5}$ cells) were seeded into the upper chamber in $200 \mu 1$ of serum-free medium and the lower compartment of the chamber was filled with $600 \mu \mathrm{l}$ DMEM supplemented with $0.2 \%$ bovine serum albumin (Gibco; Thermo Fisher Scientific, Inc.) and 10\% FBS. After incubation for $24 \mathrm{~h}$ at $37^{\circ} \mathrm{C}$, the cells on the upper surface of the membrane were carefully removed using a cotton swab. The cells that had migrated to or invaded the lower surface of the membrane were fixed in $1 \mathrm{ml}$ of $4 \%$ methanol for $10 \mathrm{~min}$ at $18-21^{\circ} \mathrm{C}$ (room temperature) and stained with $0.1 \%$ crystal violet for $30 \mathrm{~min}$ at $37^{\circ} \mathrm{C}$. A Nikon inverted microscope (TS100-F; Nikon Corporation, Tokyo, Japan) used to observe migratory cells in lower chamber (magnification, x200). 
Western blotting. MDA-MB-231 cell pellets were lysed in ice-cold radioimmunoprecipitation assay buffer containing complete protease inhibitor cocktail (Bio-Swamp, Shanghai, China) for $30 \mathrm{~min}$ on ice. The protein concentration was determined using a BCA protein assay kit. Equal amounts $(20 \mu \mathrm{g})$ of proteins were fractionated on an appropriate percentage (10\%) SDS-polyacrylamide gel and transferred onto a polyvinylidene difluoride membrane. After the transfer, non-specific binding to the membrane was blocked by incubation with $5 \%$ nonfat dry milk in Tris-buffered saline and Tween 20 (TBST) at room temperature for $2 \mathrm{~h}$, which was followed by overnight incubation with primary antibodies in TBST and 5\% nonfat dry milk on a shaker at $4^{\circ} \mathrm{C}$. The primary antibodies were as follows: Anti-MFG-E8 (1:1,000; cat. no. ab168733; rabbit); anti-E-cadherin (1:1,000; cat. no. ab76055; mouse); anti-N-cadherin (1:500; cat. no. ab18203; rabbit); anti-vimentin (1:500; cat. no. ab8978; mouse); anti-caspase-3 (1:500; cat. no. ab4051; rabbit); anti-caspase-9 (1:500; cat. no. ab69514; rabbit); anti-Bcl-2-associated $\mathrm{X}$ protein (Bax; 1:1,000; cat. no. ab53154; rabbit); anti-B-cell lymphoma 2 (Bcl-2; 1:500; cat. no. ab32124; rabbit); anti- $\beta$-actin (1:10,000; cat. no. ab8227; rabbit) (all from Abcam, Cambridge, UK); anti-cleaved caspase-3 (1:500; cat. no. 9664P; rabbit); anti-cleaved caspase-9 (1:800; cat. no. 9505P; human) (both from Cell Signaling Technology, Inc., Danvers, MA, USA); anti-matrix metalloproteinase (MMP)-9 (1:800; cat. no. sc-21733; mouse) and anti-MMP-2 (1:500; cat. no. sc-13595; mouse) (both from Santa Cruz Biotechnology, Inc., Dallas, TX, USA). The membranes were incubated with horseradish peroxidase (HRP)-conjugated secondary antibodies [Goat anti-rabbit immunoglobulin (IgG) H\&L, 1:5,000, cat. no. ab6721; goat anti-mouse IgG, 1:5,000, cat. no. ab6789; goat anti-human IgG, 1:10,000, cat. no. ab6858; Abcam] at $4^{\circ} \mathrm{C}$ overnight and the protein bands were visualized by using an Immobilon ECL Ultra Western HRP substrate (EMD Millipore, Billerica, MA, USA) and using ImageJ 1.8 software (National Institutes of Health, Bethesda, MD, USA). The levels of MMP-2 and MMP-9 were detected using an ELISA kit which was purchased from AmyJet Scientific, Inc. (cat. no. KA0391; Wuhan, China).

Flow cytometric analysis of apoptosis. The analysis of apoptotic cells was conducted using annexin V-fluorescein and PI staining. In accordance with the manufacturer's protocol (Wuhan Hualian Branch Biotechnology Co., Ltd.), transfected or untransfected control MDA-MB-231 cells were collected by trypsinization, washed with PBS, resuspended in $100 \mu 1$ annexin V FLUOS labeling solution and incubated for $10-15 \mathrm{~min}$ at $15-25^{\circ} \mathrm{C}$. Cellular apoptosis was evaluated using flow cytometry. Flow cytometric analysis clearly differentiated normal (living) cells, which exhibit low annexin $\mathrm{V}$ and low PI staining, from apoptotic cells (high annexin V and low PI staining) and necrotic cells (high annexin V and high PI staining). The data were analyzed by using CellQuest data acquisition and analysis software (version 5.1; BD Biosciences, Franklin Lakes, NJ, USA).

Statistical analysis. All data are presented as the mean \pm standard error. Statistical analysis for comparison between treated groups and corresponding controls was performed using SPSS 19.0 software (IBM Corp., Armonk,
NY, USA), and the data were analyzed using a two-sample Student's t-test or analysis of variance followed by the Least Significant Difference post hoc test. $\mathrm{P}<0.05$ was considered to indicate a statistically significant difference.

\section{Results}

MFG-E8 expression levels in breast carcinomas cell lines. RT-qPCR was used to examine the expression level of MFG-E8 in different breast carcinoma cell lines (Hs578Bst, Hs578T, MCF-7, T47D, ZR-75-3, MDA-MB-231). According to the results, the endogenous expression levels of MFG-E8 in MDA-MB-231 cells were significantly higher compared with that in other cells $(\mathrm{P}<0.01$; Fig. 1$)$. It was concluded that MFG-E8 was expressed in breast carcinoma cell lines and the expression level differed across various breast cancer subtypes.

\section{Downregulation of MFG-E8 in MDA-MB-231 cells. MFG-E8} is markedly upregulated in certain human breast carcinoma cell lines (26); however, little is known about the biological function of MFG-E8 in these cell lines. We hypothesized that MFG-E8 may play a role in breast cancer. To verify the hypothesis regarding the function of MFG-E8 in breast cancer cells, MDAMB-231 cells were selected as a model for further studies. Downregulated expression of MFG-E8 in MDA-MB-231 cells occurred following infection with lentivirus containing shRNAs, including shRNA1 and shRNA2 for MFG-E8. The mRNA expression level of MFG-E8 was reduced by 5.06 times and protein expression was reduced by 7.37 times following transfection with the lentivirus small interfering (si)RNA. RT-qPCR were used to detect the optimal shRNA to target MFG-E8. As presented in Fig. 2A, the mRNA levels of MFG-E8 were significantly decreased in the siRNA-transfected cells compared with the blank control (MDA-MB-231 untreated) and scramble control. shRNA2 was used for subsequent experiments. Western blotting, which was used to further validate the interference efficiency, revealed that the protein expression of MFG-E8 was also markedly decreased in the siRNA-transfected cells compared with the blank control-untransfected MDA-MB-231 cells and scramble control groups (Fig. 2B). These results indicated that lentivirus siRNA successfully downregulated the mRNA and protein expression of MFG-E8 in TNBC cells.

Cell viability is affected by $M F G-E 8$ in $M D A-M B-231$ cells. To explore the effect of MFG-E8 knockdown in MDAMB-231 cells, the morphological changes of MDA-MB-231 cells were observed under an inverted microscope. Morphological observation revealed that the control cells in the pSi-Scramble and untreated groups exhibited normal morphology with polygonal shapes, firmly adherent growth, distinct cell borders and intercellular tight junction. However, the MDA-MB-231 cells transfected with pSi-MFG-E8 exhibited abnormal morphology, with small and round in shape characteristics, detachment from the cell culture wells, cell debris and an increase in intercellular particles in a time-dependent manner following transfection, which are all indicative of apoptosis (Fig. 3A). These results indicated that high levels of MFG-E8 in MDAMB-231 cells is important for maintaining normal cell morphology and growth in vitro. To understand the effect 
of MGF-E8 knockdown on the cell viability, MDA-MB-231 cells were transfected with pSi-MFG-E8 for 24 to $72 \mathrm{~h}$, and evaluated using a MTT assay. As shown in Fig. 3B, MDA-MB-231 cell viability was significantly decreased in the siRNA-transfected groups compared with the blank control (MDA-MB-231 untreated) and scramble control 24, 48 and $72 \mathrm{~h}$ following transfection, while there were no significant differences between the pSi-Scramble and control groups. Taken together, the aforementioned results suggested that MGF-E8 lentivirus siRNA significantly suppressed the viability of MDA-MB-231 cells.

MFG-E8 expression affects breast cancer cell cycle. To determine whether MGF-E8 downregulation induced growth inhibition of MDA-MB-231 cells, the cell cycle distribution was evaluated by flow cytometry. The results confirmed that the cell cycles of MDA-MB-231 cells were affected by inhibition of MGF-E8, with the cell cycles being arrested at the $\mathrm{G}_{2} / \mathrm{M}$ phase. As shown in Fig. 4, the proportion of MDA-MB-231 cells that accumulated in the $\mathrm{G}_{2} / \mathrm{M}$ phase were significantly increased from $6.59 \pm 1.3 \%$ in the pSi-Scramble group and $6.21 \pm 1.9 \%$ in the blank control (MDA-MB-231 untreated) group to $26.48 \pm 1.2 \%$ in the pSi-MFG-E8 group, and reduced from $59.64 \pm 2.9$ and $57.35 \pm 3.2 \%$, respectively, to $46.42 \pm 1.6 \%$ in the $\mathrm{G}_{0} / \mathrm{G}_{1}$ phase $24 \mathrm{~h}$ after transfection. These results revealed that MDA-MB-231 cell growth inhibition were mediated by $\mathrm{G}_{2} / \mathrm{M}$ cell-cycle arrest following MGF-E8 downregulation.

$M F G-E 8$ expression regulates breast cancer cell migration and invasion. To examine the function of MFG-E8 in cell migration and invasion, migration and invasion assays were performed in 8- $\mu \mathrm{m}$ diameter pore size Transwell chambers in 24-well plates. In the Transwell migration assay, it was demonstrated that the number of cells that had migrated to the lower chambers was significantly reduced following MFG-E8 downregulation compared with the blank control (MDA-MB-231 untreated) and scramble control groups (Fig. 5A and B). Furthermore, the cell invasion assays revealed that knockdown of MFG-E8 by siRNA transfection significantly inhibited the invasion of MDA-MB-231 cells compared with the blank control (MDA-MB-231 untreated) and scramble control groups (Fig. 5A and B). To investigate the underlying mechanism of the inhibition of cell migration and invasion by MFG-E8 downregulation, western blot analysis was used to detect the expression of associated proteins. Compared with the blank control (MDA-MB-231 untreated) and scramble control groups, the expression levels of $\mathrm{N}$-cadherin and vimentin in the MFG-E8 downregulation group were markedly decreased, and the level of E-cadherin was markedly increased (Fig. 5C). In addition, the expression of MMP-9 and MMP-2 was markedly decreased, which was confirmed by the results of the ELISA, whereby MMP-2 and MMP-9 levels were significantly decreased compared with the blank control (MDA-MB-231 untreated) and scramble control groups (Fig. 5D).

Knockdown of MFG-E8 in MDA-MB-231 cells induces cell apoptosis. According to the aforementioned results, it was demonstrated that the MFG-E8 knockdown cells exhibited the typical morphology of apoptosis. Next, the proportion of apoptotic MDA-MB-231 cells under each condition was examined

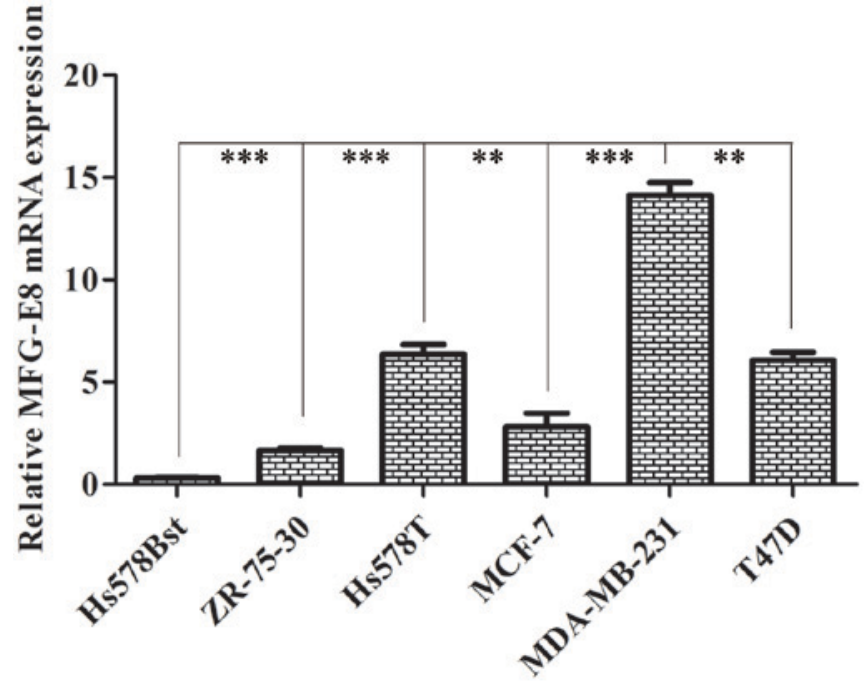

Figure 1. MFG-E8 expression level in breast carcinomas cell lines and a non-breast cancer cell line. Reverse transcription-quantitative polymerase chain reaction was used to determine the expression level of MFG-E8 mRNA in a variety of breast carcinomas cell lines and a non-breast cancer cell line. ${ }^{* *} \mathrm{P}<0.01$, MDA-MB-231 cells vs. T47D cells. ${ }^{* * *} \mathrm{P}<0.001, \mathrm{MDA}-\mathrm{MB}-231$ cells vs. MCF-7 cells. MFG-E8, Milk fat globule-EGF factor 8.

B
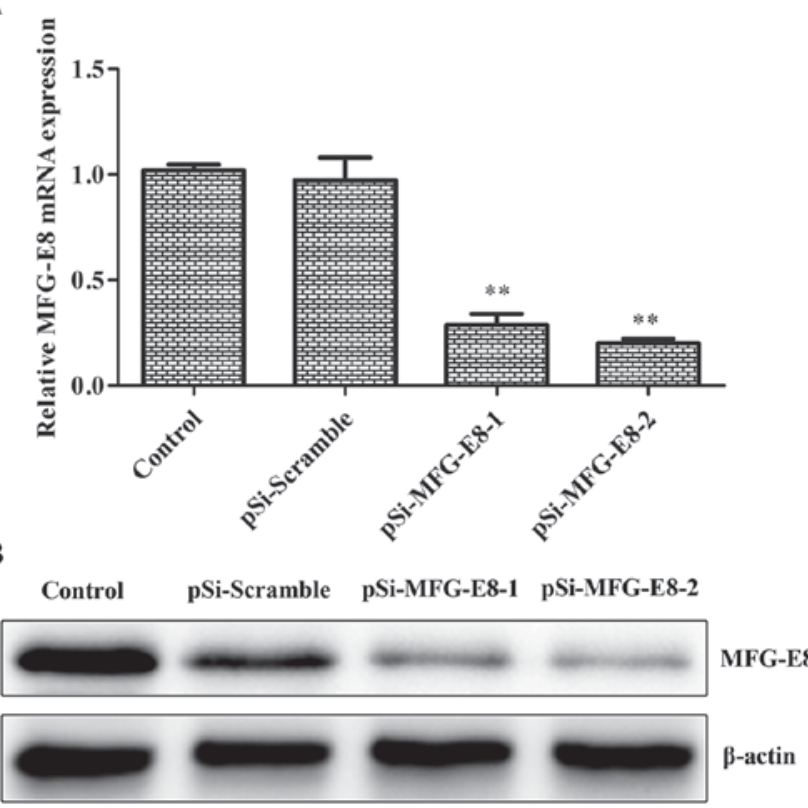

Figure 2. Downregulation of MFG-E8 in MDA-MB-231 cells. (A) Reverse transcription-quantitative polymerase chain reaction was used to determine the expression level of MFG-E8 following transfection. (B) Western blotting was used to determine the protein level of MFG-E8 after transfection. ${ }^{* *} \mathrm{P}<0.01$, pSi-MFG-E8 group vs. pSi-Scramble group. Control, non-transfected cells; pSi-Scramble, cells transfected with control shRNA lentiviral vector; pSi-MFG-E8-1, cells transfected with control shRNA1-MFG-E8 lentiviral vector; pSi-MFG-E8-2, cells transfected with control shRNA2-MFG-E8 lentiviral vector; MFG-E8, Milk fat globule-EGF factor 8; shRNA, short hairpin RNA.

using flow cytometry. MGF-E8 knockdown significantly reduced the proportion of viable MDA-MB-231 cells from $94.32 \pm 2.13 \%$ and $95.41 \pm 1.44 \%$ to $69.85 \pm 3.61 \% 48 \mathrm{~h}$ following transfection compared with the blank control (MDA-MB-231 untreated) and pSi-Scramble groups (Fig. 6A and B). At $48 \mathrm{~h}$ 
A

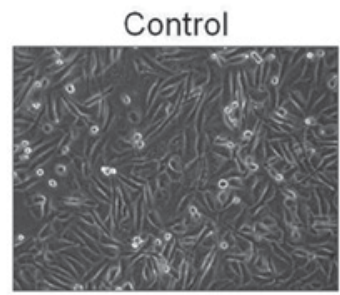
pSi-Scramble

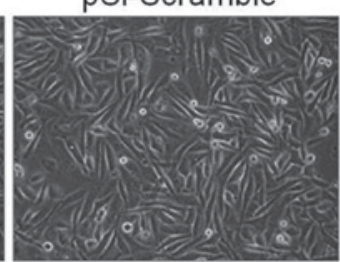

pSi-MFG-E8

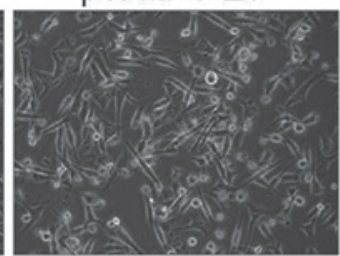

B

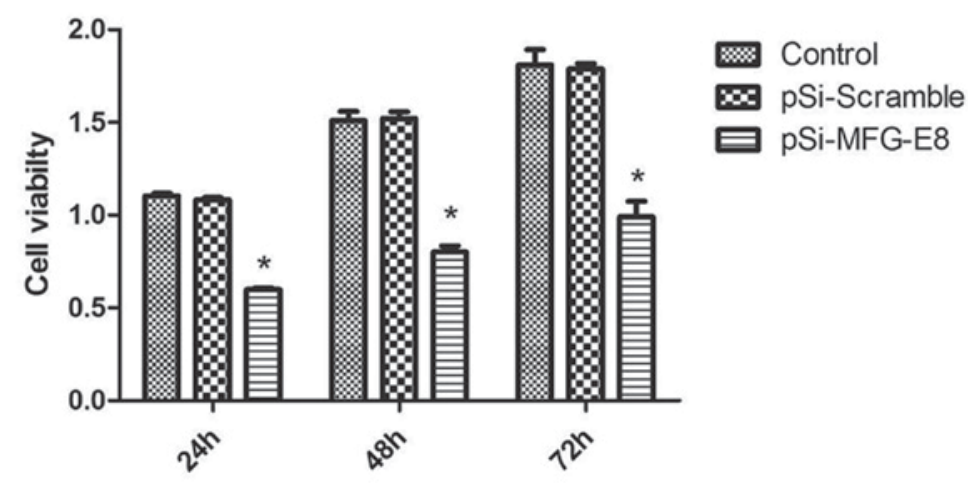

Figure 3. Cell viability is affected by MFG-E8 in MDA-MB-231. (A) The morphological changes of MDA-MB-231 cells following transfected for $48 \mathrm{~h}$ were observed under an inverted microscope (magnification, x200). (B) An MTT assay was performed to assess MDA-MB-231 cell viability at 24,48 and $72 \mathrm{~h}$ following transfection, "P<0.05, pSi-MFG-E8 group vs. pSi-Scramble group. Control, non-transfected cells; pSi-Scramble, cells transfected with control shRNA lentiviral vector; pSi-MFG-E8, cells transfected with control shRNA-MFG-E8 lentiviral vector; MFG-E8, Milk fat globule-EGF factor 8; shRNA, short hairpin RNA.

A
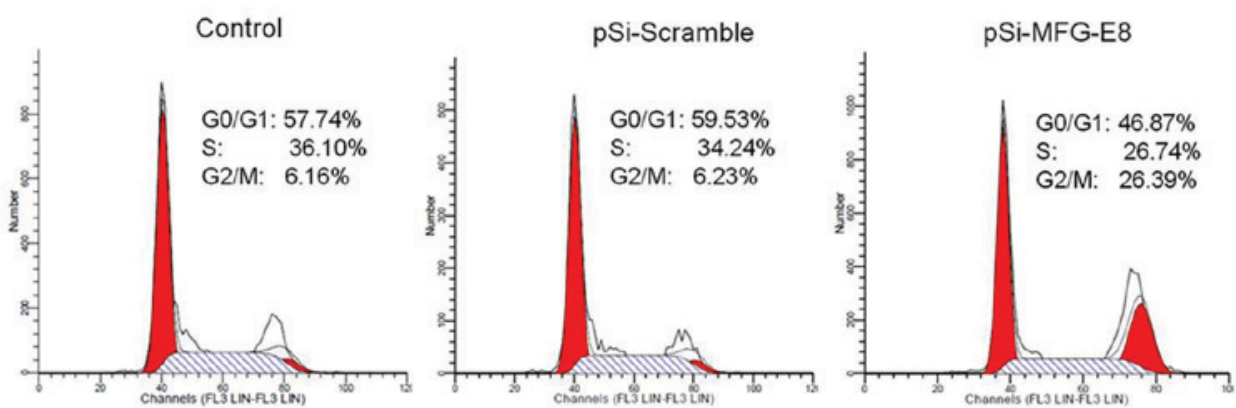

B

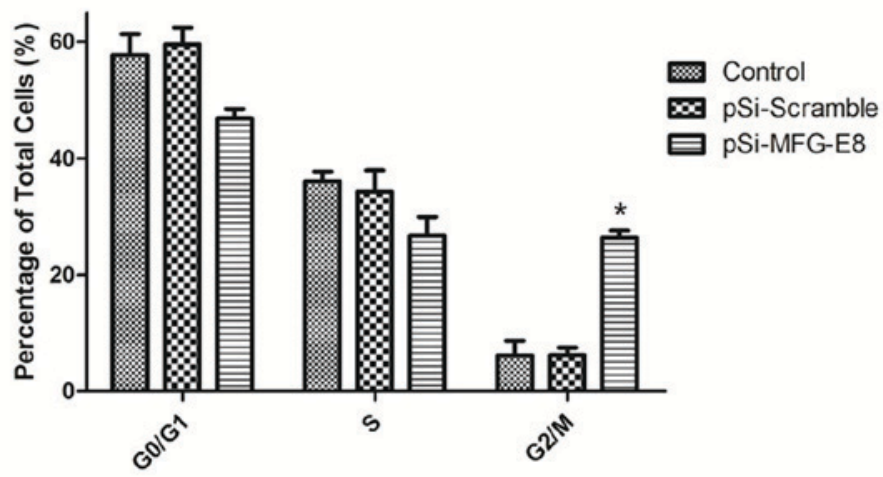

Figure 4. MFG-E8 expression affects cell cycle progression in breast cancer. (A) MDA-MB-231 cells were transfected with pSi-Scramble or pSi-MFG-E8 for $48 \mathrm{~h}$, and cell cycle distribution was measured by flow cytometry. (B) Quantification of the proportion of cells in each cell cycle phases $\left(\mathrm{G}_{0} / \mathrm{G}_{1}, \mathrm{~S}, \mathrm{G}_{2} / \mathrm{M}\right)$ presented as the mean \pm standard error. "P<0.05, pSi-MFG-E8 group vs. pSi-Scramble group. Control, non-transfected cells; pSi-Scramble, cells transfected with control shRNA lentiviral vector; pSi-MFG-E8, cells transfected with control shRNA-MFG-E8 lentiviral vector; MFG-E8, Milk fat globule-EGF factor 8; shRNA, short hairpin RNA. 
A
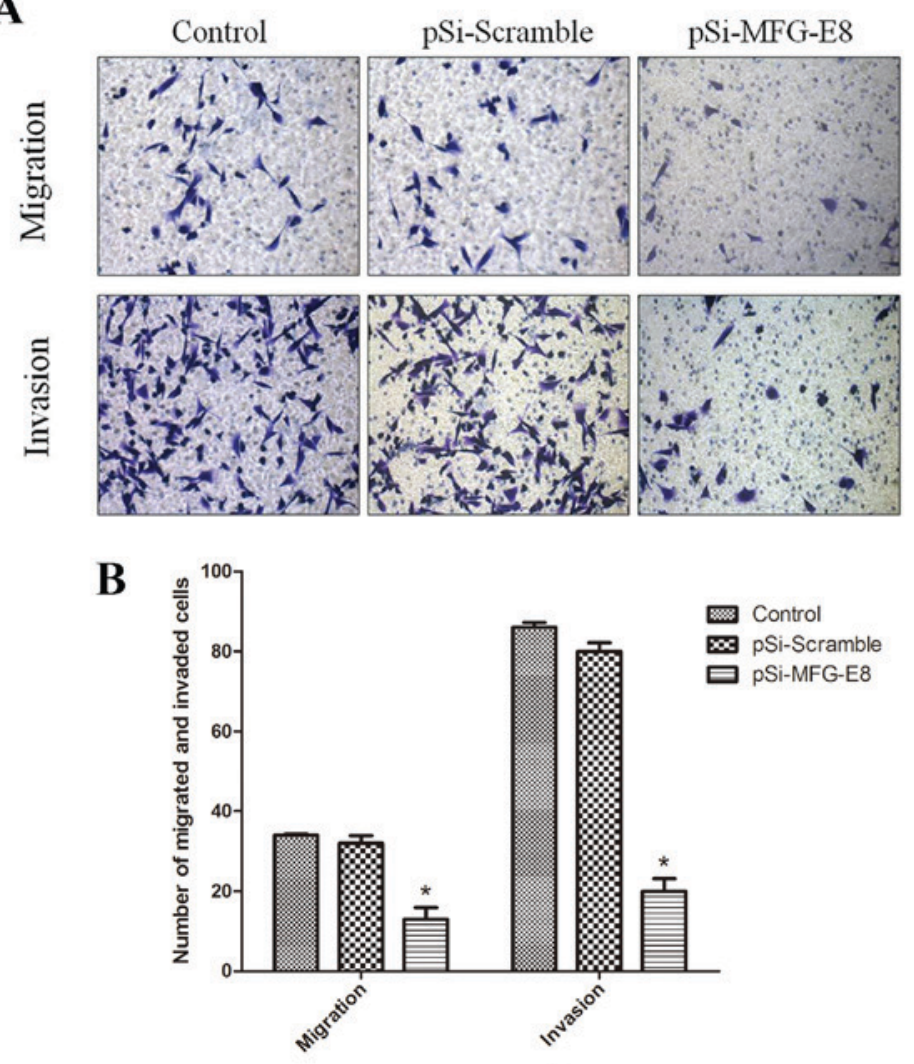

C

D
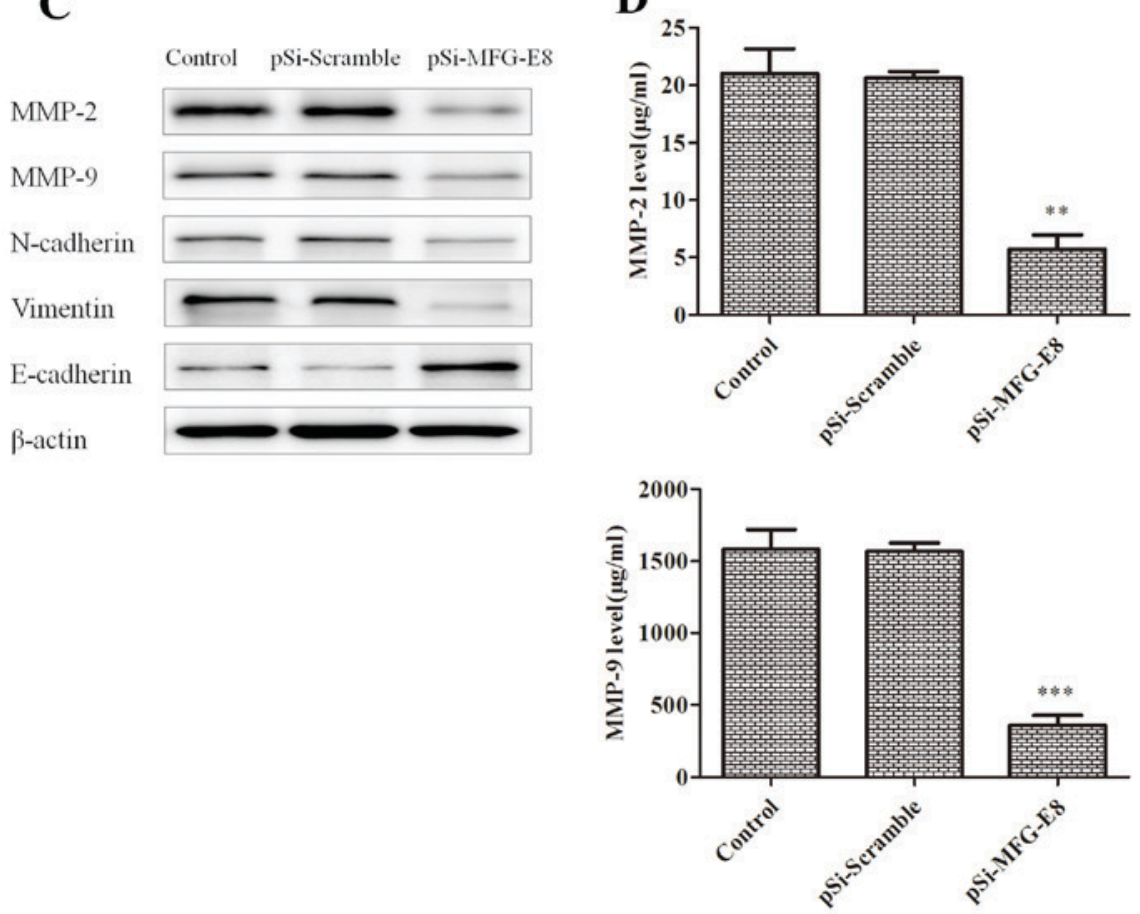

Figure 5. MFG-E8 expression regulates breast cancer cell migration and invasion. (A) Light microscopic examination following Transwell assays revealed the effects of MFG-E8 on the migration and invasion of MDA-MB-231 cells (magnification, x200). (B) The migration and invasion rate of MDA-MB-231 cells that migrated/invaded through the membrane were counted in five random fields per group. (C) Expression levels of MMP-2, MMP-9, N-cadherin, Vimentin and E-cadherin in MDA-MB-231 cells following transfection with pSi-Scramble or pSi-MFG-E8 for $48 \mathrm{~h}$ were analyzed by western blotting. (D) Levels of MMP-2, MMP-9 in MDA-MB-231 cells were analyzed by ELISA. ${ }^{*} \mathrm{P}<0.05,{ }^{* * *} \mathrm{P}<0.01,{ }^{* * * *} \mathrm{P}<0.001$, pSi-MFG-E8 group vs. pSi-Scramble group. $\beta$-actin was used as a loading control. Control, non-transfected cells; pSi-Scramble, cells transfected with control shRNA lentiviral vector; pSi-MFG-E8, cells transfected with control shRNA-MFG-E8 lentiviral vector; MFG-E8, Milk fat globule-EGF factor 8; shRNA, short hairpin RNA; MMP, matrix metalloproteinase.

post-transfection, the percentage of apoptotic MDA-MB-231 cells transfected with pSi-MGF-E8 was $24.20 \pm 1.5 \%$, significantly higher compared with that of the pSi-Scramble group
$(4.11 \pm 3.9 \%)$ and the blank control (MDA-MB-231 untreated) group $(3.76 \pm 2.2 \%)$. To confirm whether MFG-E8 knockdown affected the expression level of proteins associated with 
A
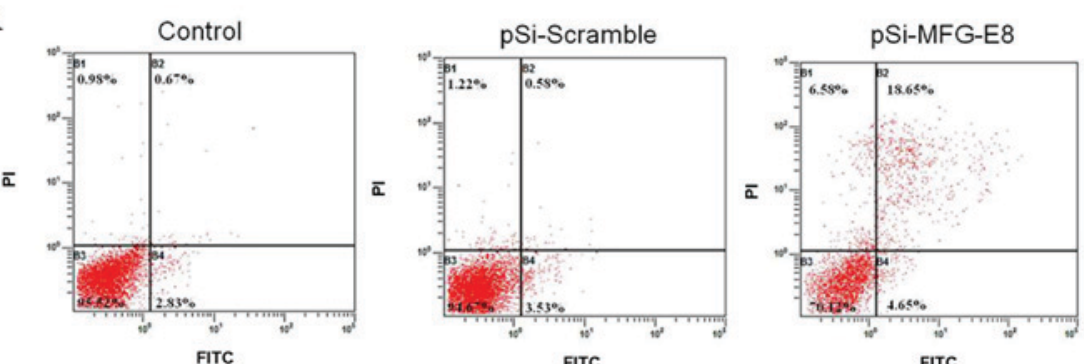

B

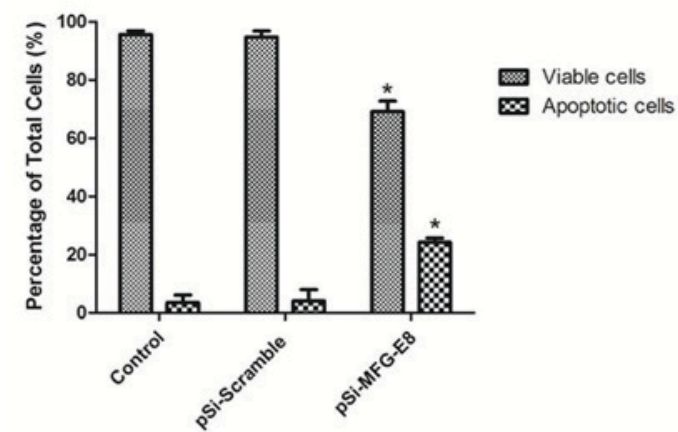

$\mathrm{C}$

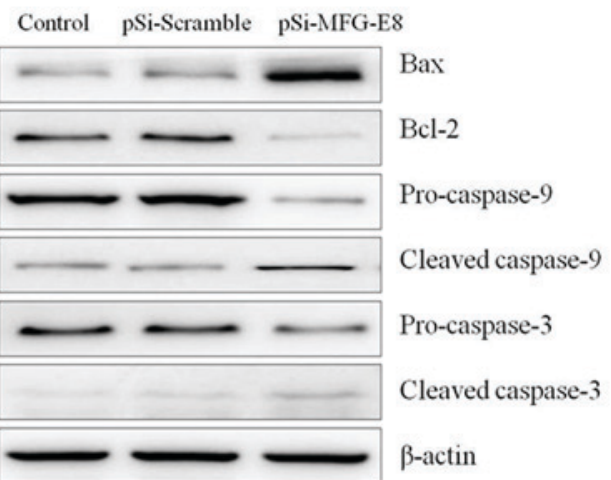

Figure 6. Knockdown of MFG-E8 in MDA-MB-231 cells induces cell apoptosis. (A) Representative plots of MFG-E8 knockdown-induced apoptosis in MDA-MB-231 cells as determined using flow cytometry. MDA-MB-231 cells were transfected with pSi-Scramble or pSi-MFG-E8, and apoptosis were evaluated by flow cytometry using FITC and PI staining $48 \mathrm{~h}$ after transfection. (B) The percentage of viable and apoptotic cells as presented as the mean \pm standard error. "P<0.05, pSi-MFG-E8 group vs. pSi-Scramble group. (C) Expression levels of caspase-9, cleaved caspase-9, caspase-3, cleaved caspase-3, Bcl-2 and Bax in MDA-MB-231 cells after transfection with pSi-Scramble or pSi-MFG-E8 for $48 \mathrm{~h}$ were analyzed by western blotting. $\beta$-actin was used as loading control. Control, non-transfected cells; pSi-Scramble, cells transfected with control shRNA lentiviral vector; pSi-MFG-E8, cells transfected with control shRNA-MFG-E8 lentiviral vector; MFG-E8, Milk fat globule-EGF factor 8; shRNA, short hairpin RNA; Bcl-2, B-cell lymphoma 2; Bax, Bcl-2-associated $\mathrm{X}$ protein; FITC, fluorescein isothiocyanate; PI, propidium iodide.

apoptosis, the protein expression levels of caspase-3, cleaved caspase-3, caspase-9, cleaved caspase-9, Bax and Bcl-2 were investigated. After MDA-MB-231 cells were transfected with pSi- MFG-E8 or pSi-Scramble for $48 \mathrm{~h}$, the expression levels of cleaved caspase-3, and cleaved caspase- 9 were markedly increased in the pSiRNA-MFG-E8 group compared with blank control (MDA-MB-231 untreated) and pSi-Scramble groups (Fig. 6C). Conversely, the expression levels of the precursor protein, caspase-3 and caspase- 9 were decreased. In addition, compared with the blank control (MDA-MB-231 untreated) and $\mathrm{pSi}$-Scramble groups, MFG-E8 downregulation markedly decreased the level of $\mathrm{Bcl}-2$ and increased the level of Bax resulting in an increase in the $\mathrm{Bax} / \mathrm{Bcl}-2$ ratio.

\section{Discussion}

Breast cancer is the most frequently diagnosed cancer and the global leading cause of cancer-associated mortality in women (1). Furthermore, it accounts for $23 \%$ of the total number of new cancer cases and $14 \%$ of the total number of cancer mortalities in the USA (31). In the past, numerous studies have examined the cellular changes in the different types of breast cancer cells. MFG-E8 is a glycoprotein that is expressed in several cell types and human malignancies (32). Previous studies have indicated that the function and expression of MFG-E8 depends on the subtype of human breast carcinoma. MFG-E8 expression is decreased in ER-positive and erbB2-positive human cancer, and may serve a suppressive function in these types. In contrast, MFG-E8 is highly expressed in TNBC cell lines and patient sera (15). However, as the expression profiles and functions of MFG-E8 in TNBC cells have not yet been thoroughly analyzed, further studies are required (33).

The aim of the present study was to explore the role of MFG-E8 in TNBC cells and examine the underlying molecular mechanisms. Western blotting and RT-qPCR 
were used to detect the expression levels of MFG-E8 in different human breast carcinoma cell lines. The gene expression data revealed that MFG-E8 was highly expressed in TNBC cells, including MDA-MB-231 (34,35), compared with in other cell lines. A MFG-E8 siRNA lentiviral vector was constructed and transfected it into MDA-MB-231 cells. It was confirmed that the expression of MFG-E8 mRNA and protein was effectively downregulated in MDA-MB-231 cells following transfection. Biological methods were used to evaluate the consequences of MFG-E8 downregulation in breast cancer cells. The morphological changes were observed using an inverted microscope that demonstrated cell shrinkage and deformation in the breast cancer MDA-MB-231 cells transfected with pSi-MFG-E8, with a time-dependent increase in the number of round, and detached cells. This was consistent with the features of apoptosis and suggested that the transfection of pSi-MFG-E8 was effective in the downregulation of MFG-E8 expression, and the induction of breast cancer cell death. The viability of MFG-E8 siRNA-transduced cells was significantly inhibited, which indicated that MFG-E8 downregulation impaired cell growth in vitro. The inhibition of MFG-E8 induced G2/M cell cycle arrest. The effect of MGF-E8 on MDA-MB-231 cell migration and invasion was assessed using a Transwell assay, and the results revealed that knockdown of MFG-E8 significantly inhibited the migration and invasion of MDA-MB-231 cells compared with the control groups. To further establish the effect of MFG-E8 on cell migration and invasion, western blotting and ELISA were used to detect the expression of migration and invasion-associated proteins. The expression of N-cadherin, vimentin, MMP-2 and MMP-9 was markedly downregulated in the MFG-E8 downregulation group, and the expression of E-cadherin was markedly upregulated compared with the control groups. In addition, the levels of MMP-9 and MMP-2 was markedly decreased, which was confirmed by the results of the ELISA compared with the control groups. The results demonstrated that the repression of MFG-E8 by siRNA significantly affected MDAMB-231 cell cycle progression and cell invasion activity via key proteins in the cell cycle and invasion associated pathway.

Apoptosis is an active process of cellular self-destruction, to explore the mechanism underlying MDA-MB-231 cell apoptosis induced by MFG-E8 downregulation, the expression of apoptosis-associated proteins were analyzed by western blotting. After MFG-E8 knockdown in MDA-MB-231 cells, the proportion apoptotic cells were significantly higher in the pSi-MFG-E8 group compared with in the $\mathrm{pSi}$-Scramble and control groups. After MFG-E8 silencing, the expression of Bax, cleaved caspase-3, and cleaved caspase-9 in the pSi-MFG-E8 group was markedly upregulated, whereas the protein expression of Bcl-2, caspase-3, and capase-9 was markedly reduced. The downregulation of caspase- 3 and the upregulation of cleaved caspase-3 provided supporting evidence of apoptosis, thus suggesting that the knockdown of MFG-E8 induced cell apoptosis through an increase in the expression of apoptosis-associated proteins. These results indicated that the MFG-E8 lentivirus siRNA decreased the viability of MDA-MB-231 cells through the induction of apoptosis. This study explored the effect of MFG-E8 on TNBC cell viability, invasion, migration and apoptosis in only one TNBC cell line, MDA-MB-231. Thus, we will further investigate the effects of MFG-E8 on proliferation, invasion, migration and apoptosis in other TNBC cell lines in subsequent studies to confirm the results of the present study.

In conclusion, MFG-E8 interference significantly suppressed the viability, migration, invasion of MDA-MB-231cells, and caused cell cycle arrest at the $\mathrm{G}_{2} / \mathrm{M}$ phase, and ultimately leading to apoptosis. The data indicated that MFG-E8 expression was significantly associated with the viability and invasive potential of TNBC. Furthermore, the inhibition of MFG-E8 may provide a novel target for the prevention and treatment of human breast carcinoma. In this study, the biological consequences of MFG-E8 downregulation in breast cancer cells were focused upon, but further in vivo and in vitro experiments are required to uncover the mechanisms of differential gene regulation in the pathogenesis of human breast carcinoma and provided potential targets associated with MFG-E8 for novel strategies for clinical treatment with human breast carcinoma.

\section{Acknowledgements}

Not applicable.

\section{Funding}

The present study was supported by a grant from the Key Scientific Research Project of Wuhan City Health and Family Planning Commission (grant no. WX16B05).

\section{Availability of data and materials}

All datasets used during the current study are available from the corresponding author on reasonable request.

\section{Authors' contributions}

YY performed the lentivirus production, oligonucleotide transfection and assessed the proliferation of cells using an MTT assay and was a major contributor in writing the manuscript. $\mathrm{JL}$ analyzed the data regarding cell proliferation, expression of associated mRNA and proteins, cell cycle, apoptosis and cell invasion activity. QS conducted the cell experiments including the expression of associated mRNA and proteins using RT-qPCR and western blotting. KZ performed cell cycle and apoptosis analysis using flow cytometry. XY performed the cell migration and invasion analysis using Transwell assay. YT contributed the conception and design of the present study. $\mathrm{JZ}$ was involved in designing the experiment protocol, all data analysis, drafting the manuscript and revising it critically for important intellectual content, giving final approval of the version to be published and was responsible for the acquisition of funding. All authors read and approved the final manuscript.

\section{Ethics approval and consent to participate}

Not applicable. 


\section{Patient consent for publication}

Not applicable.

\section{Competing interests}

The authors declare that they have no competing interests.

\section{References}

1. Jemal A, Bray F, Center MM, Ferlay J, Ward E and Forman D: Global cancer statistics. CA Cancer J Clin 61: 69-90, 2011.

2. Sung H, Ren J, Li J, Pfeiffer RM, Wang Y, Guida JL, Fang Y, Shi J, Zhang K, Li N, et al: Breast cancer risk factors and mammographic density among high-risk women in urban China. NPJ Breast Cancer 4: 3, 2018.

3. Carey LA, Metzger R, Dees EC, Collichio F, Sartor CI, Ollila DW, Klauber-DeMore N, Halle J, Sawyer L, Moore DT and Graham ML: American Joint Committee on cancer tumor-node-metastasis stage after neoadjuvant chemotherapy and breast cancer outcome. J Natl Cancer Inst 97: 1137-1142, 2005.

4. Steeg PS: Tumor metastasis: Mechanistic insights and clinical challenges. Nat Med 12: 895-904, 2006.

5. Ceriani RL, Peterson JA, Lee JY, Moncada R and Blank EW: Characterization of cell surface antigens of human mammary epithelial cells with monoclonal antibodies prepared against human milk fat globule. Somatic Cell Genet 9: 415-427, 1983.

6. Raymond A, Ensslin MA and Shur BD: SED1/MFG-E8: A bi-motif protein that orchestrates diverse cellular interactions. J Cell Biochem 106: 957-966, 2009.

7. Taylor MR, Couto JR, Scallan CD, Ceriani RL and Peterson JA: Lactadherin (formerly BA46), a membrane-associated glycoprotein expressed in human milk and breast carcinomas, promotes Arg-Gly-Asp (RGD)-dependent cell adhesion. DNA Cell Biol 16: 861-869, 1997.

8. Ogura K, Nara K, Watanabe Y, Kohno K, Tai T and Sanai Y: Cloning and expression of cDNA for O-acetylation of GD3 ganglioside. Biochem Biophys Res Commun 225: 932-938, 1996

9. Fens MH, Mastrobattista E, de Graaff AM, Flesch FM, Ultee A, Rasmussen JT, Molema G, Storm G and Schiffelers RM: Angiogenic endothelium shows lactadherin-dependent phagocytosis of aged erythrocytes and apoptotic cells. Blood 111: 4542-4550, 2008.

10. Atabai K, Fernandez R, Huang X, Ueki I, Kline A, Li Y, Sadatmansoori S, Smith-Steinhart C, Zhu W, Pytela R, et al: Mfge8 is critical for mammary gland remodeling during involution. Mol Biol Cell 16: 5528-5537, 2005.

11. Leonardi-Essmann F, Emig M, Kitamura Y, Spanagel R and Gebicke-Haerter PJ: Fractalkine-upregulated milk-fat globule EGF factor- 8 protein in cultured rat microglia. J Neuroimmunol 160: 92-101, 2005.

12. Asano K, Miwa M, Miwa K, Hanayama R, Nagase H, Nagata S and Tanaka M: Masking of phosphatidylserine inhibits apoptotic cell engulfment and induces autoantibody production in mice. J Exp Med 200: 459-467, 2004.

13. Bu HF, Zuo XL, Wang X, Ensslin MA, Koti V, Hsueh W, Raymond AS, Shur BD and Tan XD: Milk fat globule-EGF factor 8/lactadherin plays a crucial role in maintenance and repair of murine intestinal epithelium. J Clin Invest 117: 3673-3683, 2007.

14. Nandrot EF, Anand M, Almeida D, Atabai K, Sheppard D and Finnemann SC: Essential role for MFG-E8 as ligand for alphavbeta5 integrin in diurnal retinal phagocytosis. Proc Natl Acad Sci USA 104: 12005-12010, 2007.

15. Carrascosa C, Obula RG, Missiaglia E, Lehr HA, Delorenzi M, Frattini M, Rüegg C and Mariotti A: MFG-E8/lactadherin regulates cyclins D1/D3 expression and enhances the tumorigenic potential of mammary epithelial cells. Oncogene 31: 1521-1532, 2012.

16. Ceriani RL, Sasaki M, Sussman H, Wara WM and Blank EW: Circulating human mammary epithelial antigens in breast cancer. Proc Natl Acad Sci USA 79: 5420-5424, 1982.

17. Ceriani RL, Thompson K, Peterson JA and Abraham S: Surface differentiation antigens of human mammary epithelial cells carried on the human milk fat globule. Proc Natl Acad Sci USA 74: 582-586, 1977
18. Peterson JA, Zava DT, Duwe AK, Blank EW, Battifora H and Ceriani RL: Biochemical and histological characterization of antigens preferentially expressed on the surface and cytoplasm of breast carcinoma cells identified by monoclonal antibodies against the human milk fat globule. Hybridoma 9: 221-235, 1990.

19. Larocca D, Peterson JA, Urrea R, Kuniyoshi J, Bistrain AM and Ceriani RL: A Mr 46,000 human milk fat globule protein that is highly expressed in human breast tumors contains factor VIII-like domains. Cancer Res 51: 4994-4998, 1991.

20. Couto JR, Blank EW, Peterson JA and Ceriani RL: Anti-BA46 monoclonal antibody Mc3: Humanization using a novel positional consensus and in vivo and in vitro characterization. Cancer Res 55: 1717-1722, 1995 .

21. Ceriani RL and Blank EW: Experimental therapy of human breast tumors with 131I-labeled monoclonal antibodies prepared against the human milk fat globule. Cancer Res 48: 4664-4672, 1988.

22. Ceriani RL, Blank EW, Couto JR and Peterson JA: Biological activity of two humanized antibodies against two different breast cancer antigens and comparison to their original murine forms. Cancer Res 55 (Suppl 23): S5852-S5856, 1995.

23. Yamaguchi H, Takagi J, Miyamae T, Yokota S, Fujimoto T, Nakamura S, Ohshima S, Naka T and Nagata S: Milk fat globule EGF factor 8 in the serum of human patients of systemic lupus erythematosus. J Leukoc Biol 83: 1300-1307, 2008.

24. Atabai K, Jame S, Azhar N, Kuo A, Lam M, McKleroy W, Dehart G, Rahman S, Xia DD, Melton AC, et al: Mfge8 diminishes the severity of tissue fibrosis in mice by binding and targeting collagen for uptake by macrophages. J Clin Invest 119: 3713-3722, 2009.

25. Jinushi M, Nakazaki Y, Carrasco DR, Draganov D, Souders N, Johnson M, Mihm MC and Dranoff G: Milk fat globule EGF-8 promotes melanoma progression through coordinated Akt and twist signaling in the tumor microenvironment. Cancer Res 68: 8889-8898, 2008.

26. Matsuda A, Jacob A, Wu R, Zhou M, Nicastro JM, Coppa GF and Wang P: Milk fat globule-EGF factor VIII in sepsis and ischemia-reperfusion injury. Mol Med 17: 126-133, 2011.

27. Yang C, Hayashida T, Forster N, Li C, Shen D, Maheswaran S, Chen L, Anderson KS, Ellisen LW, Sgroi D and Schmidt EV: The integrin alpha(v)beta(3-5) ligand MFG-E8 is a p63/p73 target gene in triple-negative breast cancers but exhibits suppressive functions in $\mathrm{ER}(+)$ and erbB2(+) breast cancers. Cancer Res 71: 937-945, 2011.

28. Richardson AL, Wang ZC, De Nicolo A, Lu X, Brown M, Miron A, Liao X, Iglehart JD, Livingston DM and Ganesan S: X chromosomal abnormalities in basal-like human breast cancer. Cancer Cell 9: 121-132, 2006.

29. Wang X, Sang X, Diorio C, Lin SX and Doillon CJ: In vitro interactions between mammary fibroblasts (Hs 578Bst) and cancer epithelial cells (MCF-7) modulate aromatase, steroid sulfatase and $17 \beta$-hydroxysteroid dehydrogenases. Mol Cell Endocrinol 412: 339-348, 2015.

30. Livak KJ and Schmittgen TD: Analysis of relative gene expression data using real-time quantitative PCR and the 2(-Delta Delta C(T)) method. Methods 25: 402-408, 2001.

31. Perou CM, Sørlie T, Eisen MB, van de Rijn M, Jeffrey SS, Rees CA, Pollack JR, Ross DT, Johnsen H, Akslen LA, et al: Molecular portraits of human breast tumours. Nature 406: 747-752, 2000.

32. Hou L, Chen M, Zhao X, Li J, Deng S, Hu J, Yang H and Jiang J: FAT4 functions as a tumor suppressor in triple-negative breast cancer. Tumour Biol: Nov 28, 2016 (Epub ahead of print).

33. Tomao F, Papa A,Zaccarelli E, Rossi L, Caruso D, Minozzi M, Vici P, Frati L and Tomao S: Triple-negative breast cancer: New perspectives for targeted therapies. OncoTargets Ther 8: 177-193, 2015.

34. Rahman NA, Yazan LS, Wibowo A, Ahmat N, Foo JB, Tor YS, Yeap SK, Razali ZA, Ong YS and Fakurazi S: Induction of apoptosis and G2/M arrest by ampelopsin E from Dryobalanops towards triple negative breast cancer cells, MDA-MB-231. BMC Complement Alternat Med 16: 354, 2016.

35. Furuya K, Sasaki A, Tsunoda Y, Tsuji M, Udaka Y, Oyamada H, Tsuchiya $\mathrm{H}$ and Oguchi K: Eribulin upregulates miR-195 expression and downregulates Wnt3a expression in non-basal-like type of triple-negative breast cancer cell MDA-MB-231. Hum Cell 29: 76-82, 2016.

This work is licensed under a Creative Commons Attribution-NonCommercial-NoDerivatives 4.0 International (CC BY-NC-ND 4.0) License. 